\title{
Longitudinal Clinical Trial Recruitment and Retention Challenges in the Burn Population: Lessons Learned From a Trial Examining a Novel Intervention for Chronic Neuropathic Symptoms
}

Emily A. Ohrtman, BA, * ${ }^{\dagger \dagger}$ Ana Luiza Zaninotto, PhD, ${ }^{\dagger \dagger}$ Sandra Carvalho, PhD, ${ }^{\ddagger}$ Vivian L. Shie, BS, * Jorge Leite, PhD, ॥ Corinne Rose Ianni, BS, ${ }^{\dagger}$ Lewis E. Kazis, ScD, \$ Ross Zafonte, DO, I Colleen M. Ryan, MD, ${ }^{*}$ Jeffrey C. Schneider, $\mathrm{MD}^{\dagger}$ and Felipe Fregni, MD, PhD, $\mathrm{MPH}^{\dagger}$

\begin{abstract}
Long-term trials are key to understanding chronic symptoms such as pain and itch. However, challenges such as high attrition rates and poor recruitment are common when conducting research. The aim of this work was to explore these issues within a long-term randomized control trial using transcranial direct current stimulation to treat pain and itch. This parallel double blinded, placebo-controlled randomized trial was comprised of 15 transcranial direct current stimulation visits and 7 follow-up visits. Participants were over the age of 18, had a burn injury that occurred at least 3 weeks before enrollment, and reported having pain and/or itch that was moderate to severe in intensity. A total of 31 subjects were randomized into either an active or sham transcranial direct current stimulation groups. There were no significant differences between the groups in terms of age, race, education, baseline depression, or anxiety. The median dropout time was at visit 19 (visit 16 [ $S E=1.98$ ] for the sham group and visit $19[S E=1.98]$ for the active group). Analysis showed no differences in the dropout rate between groups $\left[\chi^{2}(1)=0.003, P=.954\right]$. The dropout rate was $46.7 \%$ for the sham group and $\mathbf{4 3 . 8} \%$ for the active group. Overall, $45.2 \%$ of the subjects dropped out of the trial. Long-term clinical trials are an essential part of evaluating interventions for symptoms such as chronic pain and itch. However, as seen in this trial, long-term studies in the burn population often face recruitment and adherence challenges.
\end{abstract}

Within recent years, there has been a significant decline in burn mortality rates. ${ }^{1}$ In $2016,96.7 \%$ of those treated in burn centers survived. ${ }^{2}$ Subsequently, research has started to focus on long-term outcomes and possible treatments. ${ }^{1,2}$ Chronic neuropathic pain and itch are commonly reported following burn injury. ${ }^{3,4}$ In one sample of burn survivors, over half reported having continuous pain despite being, on average, 10 years postinjury. ${ }^{5}$ Similarly, other studies have shown that itch occurs in up to $100 \%$ of children and $87 \%$ of adults with burn injuries, regardless of burn size or depth. ${ }^{4}$ Both chronic pain and itch can limit patients' daily

\footnotetext{
From the *Department of Physical Medicine and Rehabilitation, BostonHarvard Burn Injury Model System, Spaulding Rehabilitation Hospital, Harvard Medical School, Boston, Massachusetts; ${ }^{+}$Department of Physical Medicine and Rehabilitation, Spaulding Neuromodulation Center, Spaulding Rehabilitation Hospital, Harvard Medical School, Boston, Massachusetts; ${ }^{\ddagger}$ Neurotherapeutics and Experimental Psychopathology (NEP) Group, Psychological Neuroscience Lab, CiPsi, School of Psychology, University of Minho, Campus de Gualtar, Braga, Portugal; "Universidade Portucalense, Portucalense Institute for Human Development - INPP, Oporto, Portugal; \$Department of Health Law, Policy, and Management, Boston University School of Public Health, Boston, Massachusetts; ${ }^{\mathbb{I}}$ Spaulding Rehabilitation Hospital, Harvard Medical School, Boston, Massachusetts; ${ }^{* *}$ Massachusetts General Hospital, Shriners Hospitals for Children, Harvard Medical School, Boston, Massachusetts

${ }^{+}$Both authors have contributed equally to this work.

Address correspondence to Felipe Fregni, Spaulding Neuromodulation Center Spaulding Rehabilitation Hospital, 79/96 13th Street, Charlestown, MA. Email:ffregni@neuromodulationlab.org

(C) American Burn Association 2019. All rights reserved. For permissions, please e-mail: journals.permissions@oup.com.

doi:10.1093/jbcr/ivz084
}

activities, such as their employment, as well as affect quality of life..$^{3,6}$

Pain and itch have been heavily studied within the burn population. ${ }^{3-5,7}$ However, there is limited long-term research and consensus on possible treatments. Long-term pain management techniques remain similar to acute pain management and include the use of opioids, acetaminophen, antidepressants, antiepileptics, and ketamine. ${ }^{8}$ Some more conservative treatments involve massage therapy and the use of moisturizers and lotions. ${ }^{9}$ Long-term trials are key to understanding the course of chronic symptoms and their corresponding impact on overall recovery. However, long-term studies often present persistent challenges, such as high attrition rates and poor recruitment. ${ }^{10,11}$ Current research surrounding survivor outcomes postacute care is, in turn, limited. To better evaluate recovery outcomes, difficulties, and causal factors surrounding long-term research needs to be reported and analyzed.

The aim of this work was to explore the issues within a long-term randomized control trial using transcranial direct current stimulation (tDCS) to treat pain and itch. ${ }^{12}$ These results will be used as a springboard to examine the challenges of long-term clinical trial design in the burn population. The authors hypothesize that there are multifactorial barriers to study adherence that include socioeconomic, psychological, geographical, and study design factors. Examination of these factors may benefit future long-term burn outcomes research study design. Understanding these hardships is crucial to advancing effective long-term clinical trials and thus better serving the burn survivor population. 


\section{METHODS}

This study was a parallel double blinded, placebo-controlled randomized trial conducted at the Neuromodulation Center, Spaulding Rehabilitation Hospital (Charlestown, MA) through the Boston - Harvard Burn Injury Model System (2012P001996). Subjects were over the age of 18, had a burn injury that occurred at least 3 weeks before enrollment, and reported having pain and/or itch that was moderate to severe in intensity ( $\geq 4$ on the Visual Analogue Scale). Subjects were recruited through collaborating inpatient and outpatient rehabilitation hospitals. In addition, potential participants were contacted using the Partners Research Patient Data Registry (RPDR). All enrolled subjects were compensated for their time and transportation costs when necessary.

The study was split into three phases (Phases I, II, and III) and, in total, subjects were asked to complete 23 visits over 1 year. Phase I included baseline electroencephalography (EEG) and assessments, Phase II included 10 stimulation visits and 3 follow-up visits over a period of 2 months (approximately 2, 4, and 8 weeks after the last day of stimulation), and Phase III included 5 stimulation visits and 4 follow-up visits (approximately 2, 4, and 8 weeks poststimulation and 12 months after the initial baseline assessment). Participants were asked to provide informed consent at each phase to promote adherence. The data presented include all 23 visits.

During Phase 1, participants were randomly assigned to the active or sham group. In the active group, direct current was delivered for 20 minutes, with a 30 -second ramp-up and ramp-down (Soterix Medical, NY). To mimic the active condition, the sham group received stimulation for 30 seconds with the same ramp-up and ramp-down. The tDCS visits lasted approximately 30 minutes and assessment visits were 1 to 2 hours. In total, the trial was comprised of 15 stimulation visits and 7 follow-up visits.

Participants were contacted before each visit to confirm their study appointment dates and times. If a participant did not appear for their appointment, they were contacted to be rescheduled as soon as possible to remain within the follow-up time period. In the event that a participant became lost to follow-up, attempts were made to contact the participant to elucidate their reason for study withdrawal.

Independent sample $t$ tests were used to determine differences in baseline characteristics and chi-square tests for categorical variables. A $P$-value of $<0.05$ was considered statistically significant. Kaplan-Meier survival estimates were generated to analyze the median dropout time and Log-rank analysis was used to compare the dropout rate between the treatment groups.

\section{RESULTS}

A total of 34 subjects consented into Phase I of the study. Thirty-one consented into Phase II and Phase III and were randomized into either the active or sham tDCS group. There were no significant differences between the active and sham groups in terms of age, race, education, baseline depression, or anxiety. Complete demographic and clinical characteristics are presented in Table 1.
The median dropout time was at visit 19 (visit 16 $[S E=1.98]$ for the sham group and visit $19[S E=1.98]$ for the active group). The Log-rank (Mantel-Cox) analysis showed no differences in the dropout rate between groups $\left[\chi^{2}(1)=0.003, P=.954\right.$; Figure 1]. The dropout rate was $46.7 \%$ for the sham tDCS group and $43.8 \%$ for the active tDCS group. Of the participants who dropped out, reported reasons included travel time and logistics and personal issues. Overall, $45.2 \%$ of the subjects dropped out of the trial. Thibaut and Ohrtman et al detail the full results of the study. ${ }^{13}$

\section{DISCUSSION}

Long-term clinical trials are an essential part of the research process to evaluate interventions that target burn-related sequelae such as chronic pain and itch. ${ }^{3,4,7}$ However, as seen in this trial, long-term studies in the burn population face recruitment and adherence challenges.

Due to the demanding nature of long-term outcome research, most intervention studies among the burn population are short-term and in the inpatient setting. $1,16,17$ Outpatient long-term trials are harder to maintain and are uncommon. The few long-term studies in the burn population examined oxandrolone and propranolol. ${ }^{18-20}$ Some of the dropout rates were reported and, like in this study, adherence was a limitation. ${ }^{18-20}$ Overall, recruitment, retention rates, participant burn out, and travel were not frequently discussed in this literature.

Recruitment posed a particular challenge in this longterm clinical trial, despite various methods of recruitment. Mailings were sent to 1700 burn survivors identified through the authors' home institution research data registry. Nonresponders within geographic proximity of the study site received follow-up phone calls. Additionally, advertisements and flyers were circulated in inpatient and outpatient collaborating hospitals. This recruitment method does not allow for quantifying the number of recruited subjects; potential participants are required to contact study staff for participation. Of note, a recent study comparing recruitment methods found that unsolicited emails, similar to this study's mailing, had the lowest response rate, whereas face-to-face recruitment yielded the highest enrollment rate. ${ }^{21}$ In the present

Table 1. Demographic and clinical variables between groups

\begin{tabular}{lcc}
\hline & $\begin{array}{c}\text { Active tDCS } \\
(n=16)\end{array}$ & $\begin{array}{c}\text { Sham tDCS } \\
(n=15)\end{array}$ \\
\hline Age, mean $(S D)^{*}$ & $49.06(13.76)$ & $48.4(13.82)$ \\
Years of education, mean $(S D)^{*}$ & $12.5(3.08)$ & $13.1(2)$ \\
BDI at baseline, mean $(S D)^{*}$ & $11.06(9.9)$ & $14.14(11.9)$ \\
VAS Anxiety at baseline, mean & $2.6(2.8)$ & $2.1(2)$ \\
$\quad(S D)^{*}$ & $7(43.75)$ & $8(53.3)$ \\
Male, $N(\%)$ & & \\
Race/ethnicity, $N(\%)$ & $10(62.5)$ & $9(60)$ \\
White* & $5(31.25)$ & $5(33.33)$ \\
African American* & $1(6.25)$ & $1(6.67)$ \\
Hispanic or Latino* & & \\
\hline
\end{tabular}

$B D I$, Beck depression inventory; VAS, Visual Analogue Scale.

${ }^{*}$ No statistical differences between the groups $(P>0.05)$. 
Adherence per stimulation group

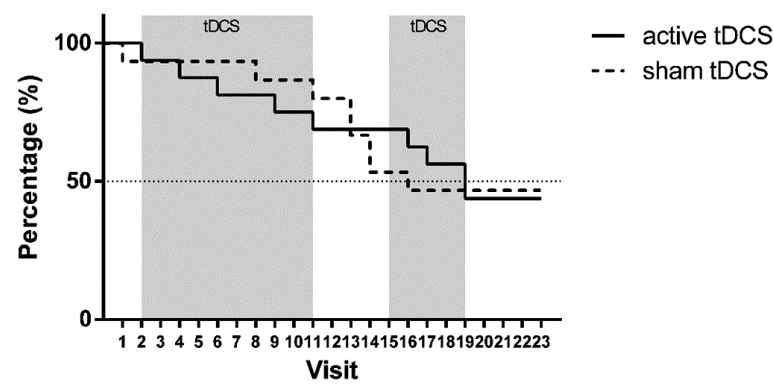

Figure 1. Log-rank survival analysis examining adherence rates in the active and sham tDCS groups. **The log-rank survival analysis is used to test the null hypothesis that there is no difference between the populations in the probability of an event (here adherence) at any time point. Adherence has been defined as the extent to which a person's behavior corresponds to the recommendations or requirements from the clinician or researcher. ${ }^{14,15}$

study, once initial contact was made with a potential participant, research staff were able to provide direct follow-up with participants.

Lower socioeconomic status is common in the burn population and may be a contributing factor to research subject participation. Research has demonstrated a higher prevalence of burn injuries in low- to middle-income communities, which may further magnify preexisting hardships given the traumatic nature of burn injuries. ${ }^{22,23}$ Previous literature has indicated that lower levels of income and education are associated with increased difficulty navigating the medical system. ${ }^{23}$ Specifically, higher education is associated with better adherence to prescribed therapy. ${ }^{24}$ Of note, in the present study, $51.6 \%$ of participants indicated their highest level of education as high school or less, whereas only $25.8 \%$ reported completion of a college degree. Lower levels of education may therefore negatively affect adherence in this study. In addition, approximately one third of participants in this study reported competitive employment. Employed participants may experience difficulties in balancing a need to earn income with time for research participation, possibly leading to recruitment challenges and sample bias. Also, study visits took place during weekday working hours which may conflict with work hours. It is also important to note that the nonworking study subjects demonstrate barriers to participation (almost two-thirds of the study subjects were not employed). Persons with socioeconomic disadvantages experience disparities in access to care and subsequent recovery as well as lack of trust in the medical system that may affect research participation. ${ }^{23,25}$ Socioeconomic disadvantages may therefore negatively affect study adherence rates.

Despite willingness to participate in research, subjects may encounter difficulties with transportation to the research location. By trying to reach a wider pool of participants, researchers often recruit from areas outside their local catchment network to create a more robust sample. However, this can negatively affect study adherence rates. Studies within other populations have previously identified transportation as a barrier to follow-up attendance, especially in urban locations. ${ }^{10}$ These barriers may include lack of transportation, limited funding for transportation, and physical impairments affecting travel ability. Compensation for transportation was offered for participants traveling locally; however, there was no funding for travel from farther areas that may require hotel and train compensation. In addition, the lack of affordable or accessible transportation is amplified by the large number of study visits (23) and served as a barrier to study recruitment and compliance.

Depression, anxiety, and posttraumatic stress disorder (PTSD) may also contribute to challenges in follow-up visits as mental health disorders are related to loss of motivation and social isolation. ${ }^{25,26,27}$ Literature has documented a high level of psychological distress at the time of burn injury and during rehabilitation. ${ }^{25,28}$ Depression and anxiety are associated with a lack of energy, demotivation, and social avoidance behaviors. ${ }^{26,29}$ Although depression and anxiety were not found to be significantly associated with dropout rates in this study, nevertheless, a number of study participants reported depression and anxiety symptoms. The Beck depression inventory (BDI) and Visual Analogue Scale (VAS) for anxiety were administered to all participants at baseline. At baseline, 29.0\% of participants had scores indicative of depressive symptoms ( $\geq 17$ on the BDI) and $41.9 \%$ of participants had scores indicative of moderate to severe anxiety symptoms ( $\geq 4$ out of 10 on the VAS anxiety). These data demonstrate preexisting mental illness symptoms in a minority of the study population. Previous studies have demonstrated that the stigma of mental illness negatively affects study recruitment and retention rates; additionally, mental illness affects one's ability to participate in a trial. ${ }^{30,31}$

The full study included 23 study visits over the course of 1 year. Previous research has demonstrated that extensive study time periods and repeated assessments cause a burden on participants and contribute to increased rates of study dropout. ${ }^{32}$ In Phase I, participants were required to attend 10 consecutive weekday study visits, likely self-selecting a higher number of unemployed and retired participants. Retirement has been associated with decreased medical adherence and may contribute in this study to decreased study adherence. ${ }^{33}$ Likewise, in a study investigating adherence to antiretroviral therapy, researchers found that nonadherence was associated with participant unemployment status. ${ }^{34}$ The lack of a daily imposed routine may contribute to increased schedule noncompliance and thus ability to maintain study adherence. ${ }^{33}$ Of note, similar to what has been shown in another tDCS trial, dropout rates for the present study are similar for both the active and sham tDCS, thus suggesting that the treatment itself was not a contributing factor for adherence in this study. ${ }^{35}$ The design of 23 study visits was therefore a contributing barrier to long-term study adherence.

This study attempted to address some of the challenges of adherence with various strategies. To combat the issue of transportation, this study reimbursed travel costs. Similar to previous research, this study also conceded one or two nonconsecutive missing visits and replaced them at the end of the daily stimulation phase. ${ }^{36}$ Using these methods in future studies may help with adherence. In addition, allowing participants to have treatments at affiliated locations, using virtual visits, or facilitating self-administration may also help. Furthermore, increased psychological care and resources postacute stay may aid recovery as well as with participation in long-term 
research. ${ }^{37}$ Other researchers have suggested obtaining multiple contact methods, including routine healthcare providers, sending duplicate mail forms, and emphasizing monetary and other study benefits as ways to increase motivation. ${ }^{10}$

Long-term clinical trials are needed to advance the care of the burn patient. It is imperative that the reasons for low adherence rates are investigated to mitigate clinical trial attrition and thereby further the evidence-based care of the long-term symptoms of burn survivors.

\section{REFERENCES}

1. Ryan CM, Schoenfeld DA, Thorpe WP, Sheridan RL, Cassem EH, Tompkins RG. Objective estimates of the probability of death from burn injuries. N Engl J Med 1998;338:362-6.

2. Emergency Department US. Burn Injury Fact Sheet. 2016

3. Schneider JC, Harris NL, El Shami A, et al. A descriptive review of neuropathic-like pain after burn injury. J Burn Care Res 2006;27:524-8.

4. Parnell LK, Nedelec B, Rachelska G, LaSalle L. Assessment of pruritus characteristics and impact on burn survivors. J Burn Care Res 2012;33:407-18.

5. Dauber A, Osgood PF, Breslau AJ, Vernon HL, Carr DB. Chronic persistent pain after severe burns: a survey of 358 burn survivors. Pain Med 2002;3:6-17.

6. Schneider JC, Bassi S, Ryan CM. Employment outcomes after burn injury: a comparison of those burned at work and those burned outside of work. J Burn Care Res 2011;32:294-301.

7. Egyhazi R, Fregni F, Bravo GL, Trinh NH, Ryan CM, Schneider JC. Chronic pain following physical and emotional trauma: the station nightclub fire. Front Neurol 2014;5:86.

8. James DL, Jowza M. Principles of Burn Pain Management. Clin Plast Surg 2017;44:737-47.

9. Anthonissen M, Daly D, Janssens T, Van den Kerckhove E. The effects of conservative treatments on burn scars: a systematic review. Burns 2016;42:508-18.

10. Grape A, Rhee H, Wicks M, Tumiel-Berhalter L, Sloand E. Recruitment and retention strategies for an urban adolescent study: lessons learned from a multi-center study of community-based asthma self-management intervention for adolescents. J Adolesc 2018;65:123-32.

11. Gul RB, Ali PA. Clinical trials: the challenge of recruitment and retention of participants. J Clin Nurs 2010;19:227-33.

12. Antal A, Terney D, Kühnl S, Paulus W. Anodal transcranial direct current stimulation of the motor cortex ameliorates chronic pain and reduces short intracortical inhibition. J Pain Symptom Manage 2010;39:890-903.

13. Thibaut A, Ohrtman EA, Morales-Quezada L, et al. Distinct behavioral response of primary motor cortex stimulation in itch and pain after burn injury. Neurosci Lett 2019;690:89-94.

14. Bland JM, Altman DG. The logrank test. BMJ 2004. 328. 1073. doi:10.1136/bmj.328.7447.1073.

15. Chakrabarti S. What's in a name? Compliance, adherence and concordance in chronic psychiatric disorders. World J Psychiatry 2014. 4: 30-6. doi:10.5498/wjp.v4.i2.30

16. Gomez M, Tushinski M, Jeschke MG. Impact of early inpatient rehabilitation on adult burn survivors' functional outcomes and resource utilization. J Burn Care Res 2017;38:e311-7.

17. Bajorek AJ, Slocum C, Goldstein R, et al. Impact of cognition on burn inpatient rehabilitation outcomes. PMR 2017;9:1-7.
18. Manzano-Nunez R, García-Perdomo HA, Ferrada P, et al. Safety and effectiveness of propranolol in severely burned patients: systematic review and meta-analysis. World J Emerg Surg 2017;12:11. doi:10.1186/ sl3017-017-0124-7

19. Ali A, Herndon DN, Mamachen A, et al. Propranolol attenuates hemorrhage and accelerates wound healing in severely burned adults. Crit Care 2015; 19:217.

20. Li H, Guo Y, Yang Z, Roy M, Guo Q. The efficacy and safety of oxandrolone treatment for patients with severe burns: a systematic review and meta-analysis. Burns. 2016;42:717-27.

21. Heerman WJ, Jackson N, Roumie CL, et al. Recruitment methods for survey research: findings from the mid-south clinical data research network. Contemp Clin Trials 2017;62:50-5.

22. Arpey NC, Gaglioti AH, Rosenbaum ME. How socioeconomic status affects patient perceptions of health care: a qualitative study. J Prim Care Community Health 2017;8:169-75.

23. Rybarczyk MM, Schafer JM, Elm CM, et al. A systematic review of burn injuries in low- and middle-income countries: epidemiology in the WHOdefined African Region. Afr J Emerg Med 2017;7:30-7.

24. Goldman DP, Smith JP. Can patient self-management help explain the SES health gradient? Proc Natl Acad Sci U S A 2002;99:10929-34.

25. Baker CP, Russell WJ, Meyer W 3rd, Blakeney P. Physical and psychologic rehabilitation outcomes for young adults burned as children. Arch Phys Med Rehabil 2007;88:S57-64.

26. Andreasen NJ, Norris AS, Hartford CE. Psychiatric complications in the severely burned. Dep Psychiatry Surg 174:785-93

27. Andreasen NJ, Norris AS, Hartford CE. Incidence of long-term psychiatric complications in severely burned adults. Ann Surg 1971;174:785-93.

28. Roh YS, Chung HS, Kwon B, Kim G. Association between depression, patient scar assessment and burn-specific health in hospitalized burn patients. Burns 2012;38:506-12.

29. Ohrtman EA, Shapiro GD, Simko LC, et al. Social interactions and social activities after burn injury: a Life Impact Burn Recovery Evaluation (LIBRE) study. J Burn Care Res 2018;39:1022-8. doi:10.1093/jbcr/ iry038.

30. Kanuch SW, Cassidy KA, Dawson NV, Athey M, Fuentes-Casiano E, Sajatovic M. Recruiting and retaining individuals with serious mental illness and diabetes in clinical research: lessons learned from a randomized, controlled trial. J Health Dispar Res Pract 2016;9:115-26. http://www. ncbi.nlm.nih.gov/pubmed/28533944.

31. Hughes-Morley A, Young B, Waheed W, Small N, Bower P. Factors affecting recruitment into depression trials: systematic review, meta-synthesis and conceptual framework. J Affect Disord 2015;172:274-90.

32. Davis LL, Broome ME, Cox RP. Maximizing retention in communitybased clinical trials. J Nurs Scholarsh 2002;34:47-53. http://www.ncbi. nlm.nih.gov/pubmed/11901967.

33. Kivimäki M, Batty GD, Hamer $M$, et al. Influence of retirement on nonadherence to medication for hypertension and diabetes. CMAJ 2013;185:E784-90

34. Semvua SK, Orrell C, Mmbaga BT, Semvua HH, Bartlett JA, Boulle AA. Predictors of non-adherence to antiretroviral therapy among HIV infected patients in northern Tanzania. PLoS One 2017;12:e189460.

35. Carvalho S, Leite J, Jones F, Morse LR, Zafonte R, Fregni F. Study adherence in a tDCS longitudinal clinical trial with people with spinal cord injury. Spinal Cord 2018;56:502-8.

36. Brunoni AR, Nitsche MA, Bolognini N, et al. Clinical research with transcranial direct current stimulation (tDCS): challenges and future directions. Brain Stimul 2012;5:175-95.

37. Goetter EM, Bui E, Ojserkis RA, Zakarian RJ, Brendel RW, Simon NM. A systematic review of dropout from psychotherapy for posttraumatic stress disorder among Iraq and Afghanistan combat veterans. J Trauma Stress 2015;28:401-9. 